\title{
Effect of Solvent Evaporation Time of Polysulfone Incorporated Copper Oxide Nanoparticles Incorporated Polysulfone Ultrafiltration Membrane on Protein Removal
}

\author{
N. S. M. Sabria, H. Hasbullah", M. S. Tohid ${ }^{\mathrm{b}}$, N. Ibrahim ${ }^{\mathrm{b}}$, R. M. Kasmani ${ }^{\mathrm{b}}$, R. R. \\ Ali $^{\mathrm{c}}$, S. A. Rahman ${ }^{\mathrm{d}}$, D. G. Fresno ${ }^{\mathrm{e}}$
}

aAdvanced Membrane Technology Research Centre (AMTEC), School of Chemical and Energy Engineering, Universiti Teknologi Malaysia, 81310 UTM Johor Bahru, Johor, Malaysia

bshool of Chemical and Energy Engineering, Universiti Teknologi Malaysia, 81310 UTM Johor Bahru, Johor, Malaysia

'Malaysia-Japan International Institute of Technology, Universiti Teknologi Malaysia Kuala Lumpur, Jalan Sultan Yahya Petra, 54100 Kuala Lumpur, Wilayah Persekutuan Kuala Lumpur, Malaysia

${ }^{\mathrm{d}}$ Faculty of Chemical \& Natural Resources Engineering, Universiti Malaysia Pahang, Lebuhraya Tun Razak, Kuantan, Malaysia eUniversidad Politécnica de Madrid, 28040 Madrid, Spain

Submitted: 19/06/2020. Revised edition: 09/07/2020. Accepted: 09/07/2020. Available online: 19/11/2020

\begin{abstract}
Polysulfone (PSf) membranes are becoming more popular in wastewater treatment recently, mostly due to its stability in chemical, thermal and mechanical properties. PSf membranes are hydrophobic, causing difficulty of water permeation. Incorporating metal oxide nanoparticles improving the membrane hydrophilicity, thus increasing membrane permeation and rejection. In this study, copper oxide nanoparticle ( $\mathrm{CuO} \mathrm{NPs}$ ) incorporated PSf membranes were fabricated under different evaporation times of $3 \mathrm{~s}, 6 \mathrm{~s}$, $8 \mathrm{~s}$, and $9 \mathrm{~s}$ to investigate on membrane morphology and performance. The membrane morphologies were characterized by using scanning electron microscope (SEM) while the membrane performance was determined through pure water flux (PWF) and bovine serum albumin (BSA) rejection. When characterized by SEM, all membranes showed an asymmetric structure with thin and dense at the top while the bottom layer was thick and porous. It was discovered that as the evaporation time increased, the formation of the finger-like structure became narrower while dense layer became thicker. When tested with PWF, membranes with higher evaporation times showed less permeability, decreasing from $139.74 \mathrm{Lm}^{-2} \mathrm{~h}^{-1}$ to $89.89 \mathrm{Lm}^{-2} \mathrm{~h}^{-1}$. In terms of BSA rejection, increased in evaporation time caused the rejection rate to increase from $87.79 \%$ to $92.15 \%$. This study proved that evaporation time is one of important parameters that influences the membrane performance significantly.
\end{abstract}

Keywords. Phase inversion, ultrafiltration, polysulfone, copper oxide nanoparticle, water treatment

\footnotetext{
* Corresponding to: H. Hasbullah (email: hasrinah@utm.my)

DOI: hhttps://doi.org/10.11113/amst.v24n3.183
} 


\subsection{INTRODUCTION}

A membrane can be defined as a semi-permeable layer that enables certain substances to pass through it while preventing others from passing. Membrane technology is a technology that has been widely in use over the past half century. There are many fields and aspects that employ the use of membrane technology, such as biotechnology, pharmaceutical, food technology, water and wastewater treatment $[1$, 2]. This technology provides a certain edge over other types of separation processes mainly because it requires a low amount of energy to operate, as no heating or cooling mediums are involved. Apart from that, membrane technology is simple to operate, requires low maintenance and is relatively cheap as compared to other types of separation methods such as distillation, absorption or extraction. Currently in wastewater treatment plant, the long-term control strategies and energy conservation are important parameter for efficient operation of wastewater treatment. These strategies can control the operating cost and also the capital cost. Membrane filtration was said to be one of the most method that can save energy and is cost efficient technologies. This technology has special attention as alternative to conventional water treatment due to ability in polishing treated wastewater effluent for reuse application [3]. The membrane technology can be further divided into several categories, which include microfiltration (MF), ultrafiltration
(UF), nanofiltration (NF), reverse osmosis (RO), and pervaporation (PV) [4].

Membrane technology, particularly ultrafiltration (UF), is widely used in protein wastewater treatment, be it industrial or domestic. UF membranes are usually fabricated from polymers. Examples of widely used polymers for UF membranes are polysulfone, polyethersulfone, polyvinylidene fluoride, cellulose acetate and polyetherimide [5]. In the recent years, polysulfone (PSf) membranes are becoming more popular for fabrication of UF membranes due to its high thermal resistance, high chemical stability and good mechanical strength as compared to other types of polymeric membranes $[1,6]$. The fabrication of UF membrane using PSf has been widely studied by previous researcher. A study by Makwana et al. [7] showed the application of PSf-PVP blend membrane for separation of oil-water mixture. They blend the membrane by using 18 wt. \% of PSf as main polymer, PVP with 5 wt.\% and adding hydrophilic nanofillers. The outcome exhibited pure water flux in range of $208-600 \mathrm{Lm}^{-2} \mathrm{~h}^{-1}$. Besides that, Sianipar et al. [8] also prepared all their UF membrane using 18 wt. \% of PSf and NMP as the solvent. The membrane produced give a result in BSA rejection about 99\%. Therefore, in this study the concentration of $18 \mathrm{wt}$ \% of PSf and NMP as a solvent was used. However, one main disadvantage of PSf membranes is that it is hydrophobic by nature, making the membrane 
more susceptible to fouling and permeation of water is more difficult [2].

In wastewater effluent, hydrophobic proteins are among the organic compounds that must be removed. Possible hydrophobichydrophobic interactions between protein and membrane will make it difficult to retain the protein because of the tendency of the protein molecules to become in proximity to the membrane surface. Worse, after some time the protein itself may end up becoming the foulant. The example of wastewater that contains protein in their effluent is come from the poultry industries. In poultry industries, protein is a major pollutant that being discharge. The continuation of disposal poultry wastewater to the environmental without following the wastewater discharge limit and guidelines may lead to harm the aquatic life and also humans. Hence, appropriate features of the ultrafiltration membrane need to be applied for treating this type of wastewater.

In order to counter this disadvantage, a lot of researchers are incorporating metal and metal oxide nanoparticles, particularly those with hydrophilic properties to increase the hydrophilicity of PSf membranes. Nanoparticles alter the morphology and the properties of polymeric membranes. Examples of metal and metal oxide nanoparticles used are $\mathrm{TiO}_{2}, \mathrm{Al}_{2} \mathrm{O}_{3}, \mathrm{ZrO}_{2}, \mathrm{SiO}_{2}, \mathrm{CuO}$ and $\mathrm{Fe}_{3} \mathrm{O}_{4}$ [9]. Depending on the type of nanoparticles used, the morphologies of polymeric membranes can be altered differently. Hence, more research is required to determine the specific effects that nanoparticles impose on different types of polymeric membranes. Study observed by Akar et al. (2013) [3] with using of $\mathrm{Cu}$ NPs in polyethersulfone (PES) membrane. Improved in membrane hydrophilicity was stated. The contact angle of PES membrane also decreases when adding of $\mathrm{Cu}$ NPs. It is supported by Baghbanzadeh et al. [10], that portrayed same result on the hydrophilicity of $\mathrm{CuO}$ NPs. These NPs lowered down the contact angle value of polyvinylidene fluoride (PVDF) membrane. Besides that, they also reported increases in water flux compared to the neat PVDF membrane. The concentrations of NPs added in membrane has played a vital role in effecting membrane performance and characteristic. When the membrane incorporated with NPs beyond the optimum loading, the performance getting worsen [11]. Nasrollahi et al. [12] measured the release of copper ion from 0.2 wt. $\%$ of $\mathrm{CuO} / \mathrm{PES}$ ultrafiltration membrane. After $120 \mathrm{~h}$ from immersion, the released content of $\mathrm{CuO}$ nanoparticle in surrounding media was $\leq 5 \mathrm{ppb}$. They also stated that $\mathrm{CuO}$ nanoparticles or $\mathrm{Cu} 2+$ ions were hardly leach from the PES membrane structure and the membrane water flux performance.

The evaporation time during casting has an effect on the morphology of polymeric membranes. According to a certain research, it is found that an increase in evaporation time will result the formation of the bottom layer support structure of 
cellulose acetate to be more compact, which reduces the permeability of the membrane [13]. The same research also indicated that increasing the evaporation time also reduces the membrane pore size, thus increasing the selectivity of membranes. Another research conducted proved that by increasing the evaporation time give a similar result with using aromatic polyamidohydraozide membranes [14].

As such, this study focuses on the effect of evaporation time in terms of membrane morphology, membrane performance specifically for pure water flux (PWF) and rejection of bovine serum albumin (BSA). Furthermore, not much study about the casting evaporation step has been made on the application of nanoparticles especially the using of $\mathrm{CuO}$ NPs incorporated PSf polymeric membranes for wastewater treatment.

\subsection{METHODS}

\subsection{Materials}

The materials used in this study were mainly for fabrication of the $\mathrm{PSf} / \mathrm{CuO}$ incorporated polymeric membranes. All of the materials used are analytical grade and are suited for laboratorial use. Copper oxide $(\mathrm{CuO})$ was obtained in size that is less than $50 \mathrm{~nm}$ from Sigma Aldrich. The Nmethyl-2-pyrrolidone (NMP) which is used as the solvent for dope solution by Merck has purity of 99.5\% and molecular weight of 99.13 $\mathrm{g} / \mathrm{mol}$. The polyvinnylpyrrolidone
(PVP) was used as a pore former and PSf (Udel-P1700) was from Solvay Advanced Polymers that comes in pallets with average molecular weight of $67,000-72,000 \mathrm{~g} / \mathrm{mol}$. Bovine serum albumin (BSA) was purchased from Sigma Aldrich of $>98 \%$ purity and is used in BSA rejection test. Glycerol, with molecular weight of $92.1 \mathrm{~g} / \mathrm{mol}$, was obtained from Merck.

\subsection{Dope Solution Preparation}

The dope solution for PSf incorporated with $\mathrm{CuO}$ nanoparticles were prepared with the composition shown in Table 1. Firstly, 76.85 wt. $\%$ of N-methyl-2-pyrrolidone (NMP) was weighed and poured into a scotch bottle. Then, 0.15 wt. $\% \mathrm{CuO}$ nanoparticles were weighed on a weighing paper and added to the scotch bottle. The solution was then left to sonicate for 1 hour at $25^{\circ} \mathrm{C}$ to ensure thorough mixing. After sonicating for 1 hour, 5 wt. \% pore former polyvinylpyrrolidone (PVP) was weighed and added to the scotch bottle. A magnetic stirrer was then added and the scotch bottle was rested on a mixing setup to stir at 20 $\mathrm{rpm}$ and $50^{\circ} \mathrm{C}$ by leaving it to stir for 2 hours. After that, main polymer with 18 wt. \% polysulfone (PSf) was weighed and half of it was added to the scotch bottle while mixing. When the PSf has started to dissolve, another half of PSf was added and the solution was left to stir for one full day to obtain a homogenous solution. Finally, the mixing setup was removed and the scotch bottle is left to be degassed for one day. 
Table 1 Casting conditions of $\mathrm{PSf} / \mathrm{CuO}$ membrane

\begin{tabular}{|l|l|}
\hline Conditions & Details \\
\hline Process & $\begin{array}{l}\text { Dry-wet phase } \\
\text { inversion process }\end{array}$ \\
\hline Evaporation time $(\mathrm{s})$ & $3-9$ \\
\hline Coagulation bath & Water \\
\hline $\begin{array}{l}\text { Coagulation bath } \\
\text { temperature }\left({ }^{\circ} \mathrm{C}\right)\end{array}$ & 25 \\
\hline $\begin{array}{l}\text { Ambient } \\
\text { temperature }\left({ }^{\circ} \mathrm{C}\right)\end{array}$ & Room Temperature \\
\hline $\begin{array}{l}\text { Membrane casting } \\
\text { thickness }(\mu \mathrm{m})\end{array}$ & 0.12 \\
\hline
\end{tabular}

\subsection{Membrane Fabrication}

The fabrication of flat sheet membrane was casted using phase inversion via immersion precipitation process. All of the equipment to be used, namely the glass rod and glass base, was to be thoroughly cleaned and wiped dry before each casting process. A basin of water was prepared beforehand as the coagulation bath. The detail of casting conditions was listed in Table 1.

Firstly, the dope solution was poured along the top edge of the glass base. A glass rod was then used to roll the solution from the top to the bottom of the glass base. The stopwatch was started and the solution was left to be evaporated at different evaporation times $(0 \mathrm{~s}, 3 \mathrm{~s}, 6$ s, $8 \mathrm{~s}$, and $9 \mathrm{~s}$ ). After each respective immersion times, the glass base was immersed into the basin of water for immersion precipitation process to take place. After casting, the membranes were soaked with tap water for two days in order to get rid of excess solvent on the membrane. The water was changed daily. After that, the membrane was immersed in $10 \%$ glycerol solution for another day. The 10\% glycerol solution was prepared by mixing $900 \mathrm{ml}$ ultrapure water and $100 \mathrm{ml}$ of glycerol. After that, the membranes were hung to dry at room conditions and stored in a sealed plastic bag prior to be testing.

\subsection{Membrane Characterizations}

\subsubsection{Scanning Electron Microscopy (SEM)}

The morphologies of all the membranes were inspected by using scanning electron microscope (SEM). The model of the SEM was HITACHI Table Top TM3000. The sample for SEM must be prepared beforehand. The membranes were fractured in liquid nitrogen to obtain a clear and smooth cross-section, before sticking onto a metal plate using double-sided tape at lateral sides. The plate was then inserted into the SEM and images were captured.

\subsection{Membrane Performance}

\subsubsection{Pure Water Flux}

Pure water flux of flat sheet membranes was measured by using a cross flow ultrafiltration system. The system uses a diaphragm pump to supply pressurized water that will force water through a membrane with an effective surface area of 23.76 $\mathrm{cm}^{2}$, which was cut into a circle shape and set up into the system. Water was then flowed and 
pressurized through the membrane at 2 bar for 30 minutes in order to obtain stable flow. After that, the actual permeate was measured for every 10 minutes under 1 bar of water pressure and repeated three times to obtain average value. The pure water flux was calculated using the following equation:

$J_{w}=\frac{Q}{\Delta t A}$

where $J_{\mathrm{w}}$ is the pure water flux $\left(\mathrm{Lm}^{-}\right.$ $\left.{ }^{2} \mathrm{~h}^{-1}\right)$. $Q$ is the volume of permeate (L), $\Delta t$ is the permeation time (h) and $A$ is the membrane effective surface area $\left(\mathrm{m}^{2}\right)$.

\subsubsection{Filtration of Protein}

The protein rejection tests which measure the separation performance of the membrane. Bovine Serum Albumin (BSA) is a type of protein that is often used to measure the rejection rate of membranes. The equipment used was the same as the one used for water permeation, which is the cross-flow ultrafiltration system. The BSA solution was first prepared beforehand by mixing $0.5 \mathrm{~g}$ of BSA powder to $1000 \mathrm{~mL}$ of pure water, where a $500 \mathrm{mg} / \mathrm{L}$ of BSA solution was formed. The experiment was run under 1 bar of pressure and the BSA solution as feed solution. 10 $\mathrm{mL}$ of permeate was collected for each membrane sample and the time taken to collect $10 \mathrm{~mL}$ of permeate was recorded. The concentration of BSA in the feed and permeate were determined using UV-Vis Spectrometer (DR 5000, Hach) at a single absorbance wavelength of 280 $\mathrm{nm}$. Then the BSA rejection was obtained by calculating using the following equation:

rejection $\%=\left(1-\frac{C p}{C f}\right) x 100$

where $C p$ and $C f$ are the BSA concentration in the permeate and in the feed, respectively.

\subsection{RESULTS AND DISCUSSION}

\subsection{Membrane Morphology}

The morphologies of polymeric membranes are highly affected by the evaporation time during membrane fabrication. Different evaporation times have led to different membrane structures and morphologies. Figure 1 shows the SEM images of the crosssections of membranes with different evaporation times. All the membranes in this study achieved an asymmetric structure consisting of a thin dense layer at the top and a porous layer at the bottom part of each membrane. The bottom layer consists of many finger-like structures that are also known as macrovoids [15].

When observing the possible trends in the SEM images, it can be seen that an increase in evaporation time resulting in thicker dense layer at the top. When a casted membrane solution immersed in a coagulation bath, phase inversion occurs initially at the top of the membrane sheet, which results in high concentration gradient of the polymer, from bottom 
to top. When the evaporation time of a membrane was increased, the amount of volatile solvents at the top of the membrane that lost due to increased evaporation. Thus, the formation of a more concentrated polymer region at the top of the membrane, making the thin, dense layer thicker as the evaporation time
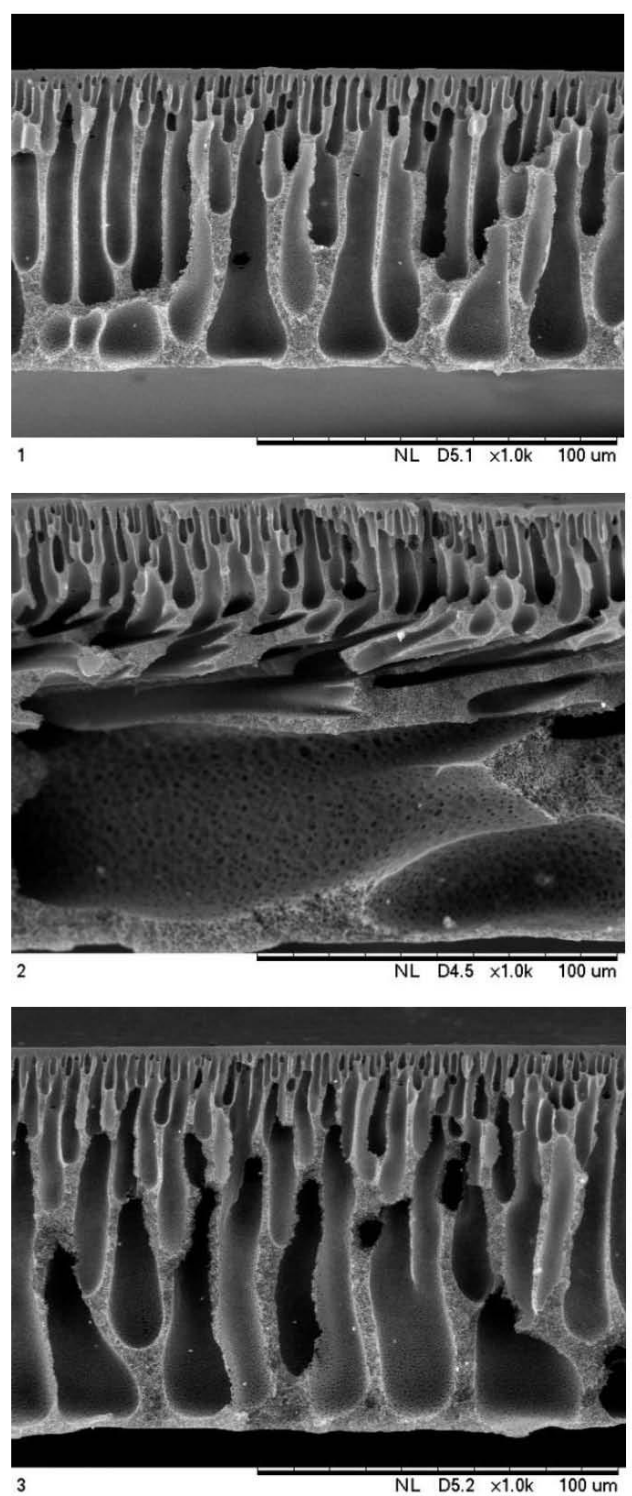

increased [13]. This type of nascent skin layer had high resistance to mass transfer of solvents and non-solvents between the coagulation bath and the interior region of membrane during the wet phase inversion process. Formation of this resistive top layer caused a delayed phase separation for solidification in sub-layer.

$3 s$

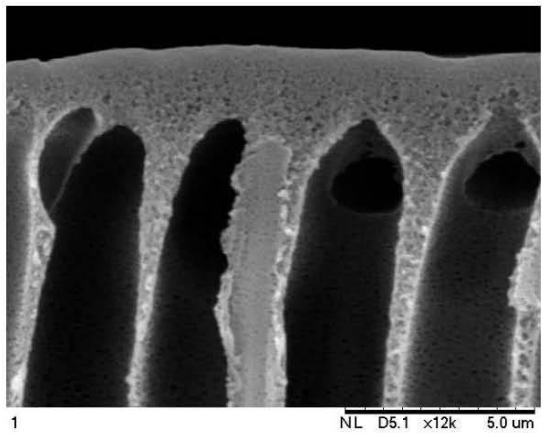

$6 s$

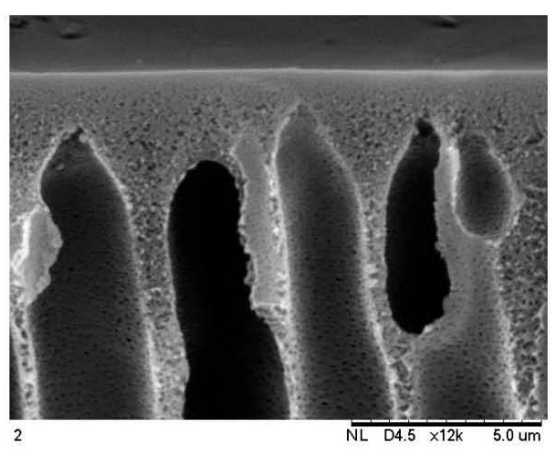

$9 \mathrm{~s}$

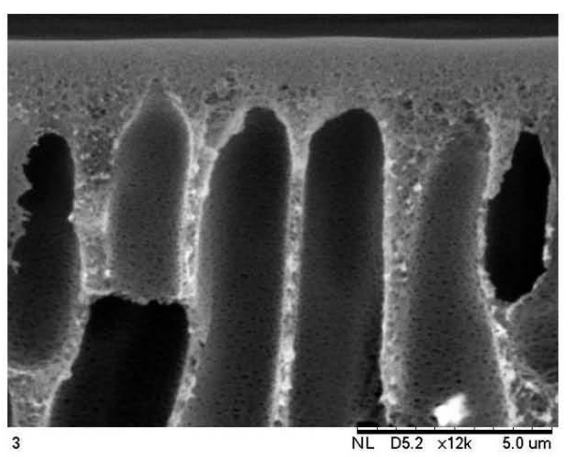

Figure 1 Cross-section view of SEM images at $1.0 \mathrm{~K}$ (left) and 12.0K (right) magnification at different evaporation times 
By analyzing the morphologies, it was observed that as the evaporation time increased, the size of the fingerlike structures became shorter and also decreased. This observation tallies with findings that mentioned the shrinkages of the pores are can be explained by the kinetic aspect of the formation of membrane by phase inversion technique. In case of a fast demixing process with little evaporation time, membrane pores that were formed immediately induced a porous, wide finger-like structures [16]. Image from SEM proved that, finger-like structure at top layer of membrane become narrower as evaporation time increase. Another research supported and described the same effect occurred for cellulose acetate (CA) membranes. The research described that macrovoids are suppressed and will appear thinner when the duration of the evaporation time is increased enough [17].

\subsection{Water Flux Performance}

Pure water flux is used to measure the permeability of membranes. A membrane that is suited for real life applications must be highly permeable to the components that can pass through it which, in this scenario, is water. As such, in order to increase membrane permeability, the membrane must possess waterloving properties, or better known as hydrophilic properties [9]. Thus, it is essential to understand that evaporation time plays a large role in order to display better hydrophilic membrane properties. The results for pure water flux and in this study is displayed in Figure 2.

When observing the graph, as the evaporation time increased, the pure water flux decreased. This corresponded to the morphology trend of the polymeric membranes. As the evaporation time increased, the thin, dense layer at the top of the membrane becomes thicker and denser, making the layer less porous and causing difficulty for water to permeate through which, in turn, reduced the water flux [16]. The evaporation step in the membrane preparation procedure create high retentive membrane which lead to formation of dense membrane and slow down the flux performance. 


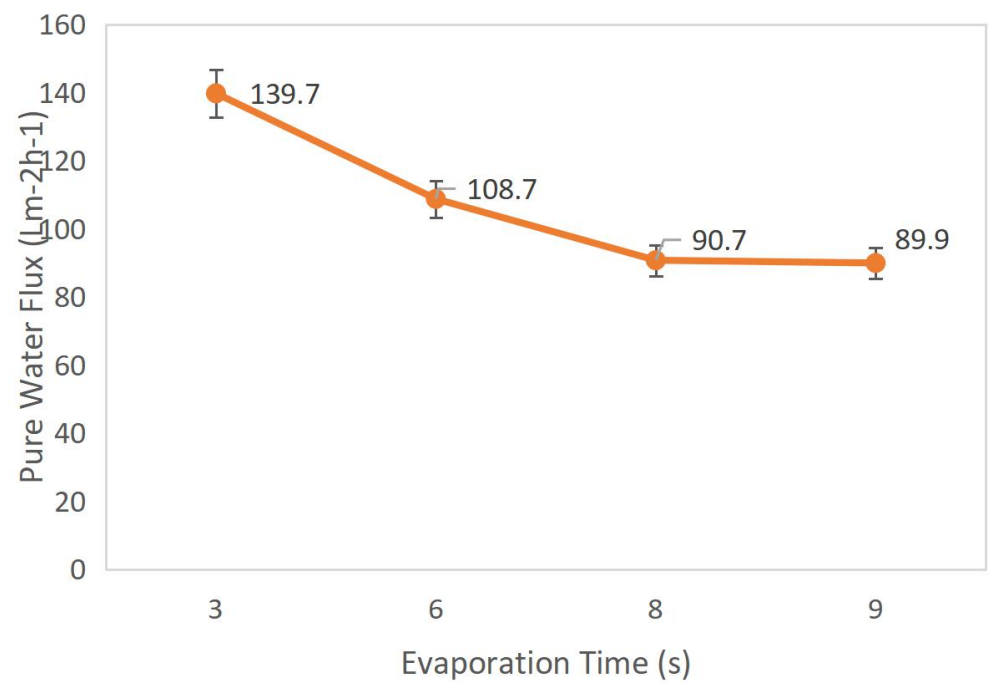

Figure 2 PWF analysis on membranes with different evaporation times

\subsection{Protein Removal Performance}

In order to test the selectivity of a membrane, the rejection rate of each membrane must be analyzed. In this study, bovine serum albumin (BSA) was used as the solute that will be rejected by the membrane. Figure 3(a) displays the graph of BSA rejection rate against evaporation time, while Figure 3(b) shows the graph of BSA flux against evaporation time.

Based on Figure 3(a), as the evaporation time increases, the rejection rate also increases. Tis relate to the membrane morphology, when the evaporation time of PSf membrane increased, the pore size and the macrovoids of the membrane became smaller, as well as making the membrane dense layer thicker. These conditions caused the BSA solute to be retained and rejected by the layer, causing higher rejection rate. This result was aligned to the findings from Kusworo et al. that also obtained similar results when testing with cellulose acetate membrane [18].

Apart from that, when observing the time taken to collect BSA sample, the time taken to collect BSA sample was directly proportional to the evaporation time of membranes. This meant that the BSA flux decreased as the evaporation time increased as shown in Figure 3(b). This justified with the same reason as the water permeation, where thicker dense top layer of membrane reduced the flux and permeation. 


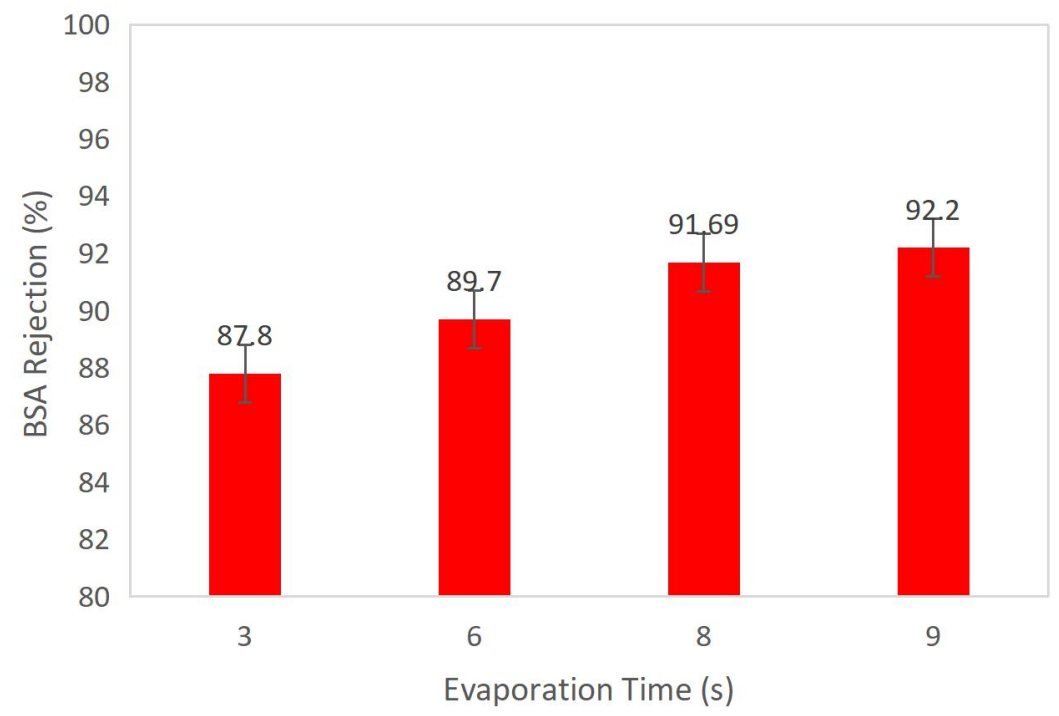

(a)

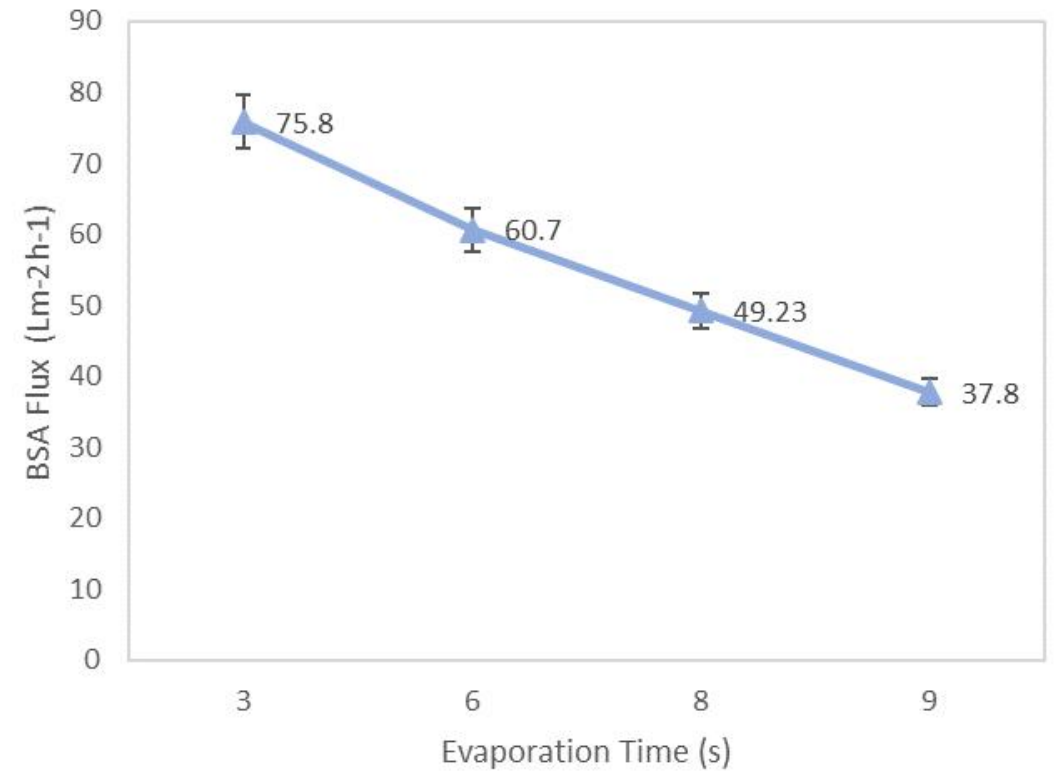

(b)

Figure 3 (a) BSA rejection $\%$ and (b) BSA flux against evaporation time

\subsection{CONCLUSION}

In this study, $\mathrm{CuO}$ incorporated polysulfone membranes were fabricated with different casting evaporation times. It was found that the evaporation time during the casting process affected the morphologies and performance of PSf membranes significantly. In terms of membrane morphology, increasing the evaporation time decreased and narrowed the size of finger-like structure as well as causing an increase in the thickness of the dense top layer in the membrane. In terms of membrane performance, it was discovered that the water permeation was inversely proportional to the evaporation time, while the rejection rate was directly proportional to the evaporation time. 


\section{ACKNOWLEDGEMENT}

The authors gratefully acknowledge financial support from several parties, namely, the Malaysia Ministry of Education (MOE) through Higher Institution Centre of Excellence (HICoE) Research Grant (4J206), Universiti Teknologi Malaysia (UTM) through Research University grant (16H10, 18H34) and UTMShine (09G17). Appreciation also goes to UTM Research Management Centre for both financial and technical support.

\section{REFERENCES}

[1] Z. Xu, S. Ye, Z. Fan, F. Ren, C. Gao, Q. Li, G. Li and G. Zhang. 2015. Preparation of $\mathrm{Cu} 2 \mathrm{O}$ Nanowire-blended Polysulfone Ultrafiltration Membrane with Improved Stability and Antimicrobial Activity. J Nanopart Res. 17: 409.

[2] Z. Xu, S. Ye, G. Zhang, W. Li, C. Gao, C. Shen and Q. Meng. 2016.

Antimicrobial

Polysulfone Blended

Ultrafiltration Membranes

Prepared with Ag/Cu2O Hybrid

Nanowires. Journal of Membrane Science. 509: 83-93.

[3] N. Akar, B. Asar, N. Dizge, I. Koyuncu. 2013. Investigation of Characterization and Biofouling Properties of PES Membrane Containing Selenium and Copper Nanoparticles. Journal of Membrane Science. 437: 216226.

[4] Baker. 2004. Membrane Technology and Applications.
John Wiley \& Sons, McGrawHill. 3: 590.

[5] X. Y. Chen, H. Vinh-Thang, A. A. Ramirez, D. Rodrigue and S. Kaliaguine. 2015. Membrane Gas Separation Technologies for Biogas Upgrading. RSC Advances. 5: 24399.

[6] H. L. Richards, P.G. L. Baker and E. Iwuoha. 2012. Metal Nanoparticle Modified Polysulfone Membranes for Use in Wastewater Treatment: A Critical Review. Journal of Surface Engineered Materials and Advanced Technology. 2: 183-193.

[7] D. Makwana, V. Polisetti, J. Castaño, P. Ray, H. C. Bajaj. 2020. Mg-Fe Layered Double Hydroxide Modified Montmorillonite as Hydrophilic Nanofiller in PolysulfonePolyvinylpyrrolidone Blend Ultrafiltration Membranes: Separation of Oil-water Mixture. Applied Clay Science. 192: 105636.

[8] M. Sianipar, S. H. Kim, C. Min, L. D.Tijing, H. K. Shon. 2016. Potential and Performance of a Polydopamine-coated

Multiwalled Carbon nanotube/polysulfone

Nanocomposite Membrane for Ultrafiltration Application. Journal of Industrial and Engineering Chemistry. 34: 364-373.

[9] M. Homayoonfal, M. R. Mehrnia, Y. M. Mojtahedi and A. F. Ismail. 2013. Effect of Metal and Metal Oxide Nanoparticle Impregnation 
Route on Structure and Liquid Filtration Performance of Polymeric Nanocomposite Membranes: A Comprehensive Review. Desalination and Water Treatment. 51: 32953316.

[10] M. Baghbanzadeh, D. Rana, C. Q. Lan and T. Matsuura. 2016. Effects of Inorganic NanoAdditives on Properties and Performance of Polymeric Membranes in Water Treatment. Separation and Purification Reviews. 45(2): 141-167.

[11] I. Sawada, R. Fachrul, T. Ito, Y. Ohmukai, T. Maruyama and $\mathrm{H}$. Matsuyama. 2012. Development of a Hydrophilic Polymer Membrane Containing Silver Nanoparticles with Both Organic Antifouling and Antibacterial Properties. Journal of Membrane Science. 387-388: 1-6.

[12] N. Nasrollahi, S. Aber, V. Vatanpour and N. M. Mahmoodi. 2019. Development of Hydrophilic Microporous PES Ultrafiltration Membrane Containing $\mathrm{CuO}$ Nanoparticles with Improved Antifouling and Separation Performance. Materials Chemistry and Physics. 222: 338-350.

[13] W. N. R. Jami'an, H. Hasbullah, F. Mohamed, N. Yusof, N. Ibrahim and R. R. Ali. 2016. Effect of Evaporation Time on Cellulose Acetate Membrane for Gas Separation. IOP Conf. Series: Earth and Environmental Science. 36: 1-5.
[14] K. Chan, T. Matsuura and S. Sourirajan. 1984. Effect of Evaporation Time on Pore Size and Pore Size Distribution of Aromatic Polyamidohydrazide RO/UF Membranes. Ind. Eng. Chem. Prod. Res. Dev. 23: 492500.

[15] A. Abdelrasoul, H. Doan, A. Lohi and C. Cheng. 2014. Morphology Control of Polysulfone Membranes in Filtration Processes: A Critical Review. Chem. Bio Eng. Rev. 2(1): 22-43.

[16] I. Syahbanu, B. Piluharto, S. Khairi and Sudarko. 2017. Effect of Evaporation Time on Separation Performance of Polysulfone/Cellulose Acetate (PSf/CA) Membrane. IOP Conf. Series: Material Science and Engineering. 299: 012040.

[17] F. G. Paulsen, S. S. Shojaie and W. B. Krants. 1994. Effect of Evaporation Step on Macrovoid Formation in Wet-cast Polymeric Membranes. Journal of Membrane Science. 91: 265282.

[18] T. D. Kusworo, Budiyono, D. Ikhsan, N. Rokhati, A. Prasetyaningrum, F. R. Mutiara and N. R. Sofiana. 2017. Effect of Combination Dope Composition and Evaporation Time on the Separation Performance of Cellulose Acetate Membrane for Demak Brackish Water Treatment. MATEC Web of Conferences. 101: 01004. 